\title{
Human pDCs preferentially sense enveloped hepatitis A virions
}

\author{
Zongdi Feng, ${ }^{1}$ You Li, ${ }^{1}$ Kevin L. McKnight,, ${ }^{1}$ Lucinda Hensley, ${ }^{1}$ Robert E. Lanford, ${ }^{2}$ Christopher M. Walker, ${ }^{3}$ and Stanley M. Lemon ${ }^{1,4,5}$ \\ ${ }^{1}$ Lineberger Comprehensive Cancer Center, The University of North Carolina at Chapel Hill, Chapel Hill, North Carolina, USA. ${ }^{2}$ Southwest National Primate Research Center, Texas Biomedical Research Institute, \\ San Antonio, Texas, USA. 'enter for Vaccines and Immunity, The Research Institute at The Nationwide Children's Hospital, and Department of Pediatrics, College of Medicine, The Ohio State University, \\ Columbus, Ohio, USA. ${ }^{4}$ Division of Infectious Diseases, Department of Medicine, and ${ }^{5}$ Department of Microbiology and Immunology, The University of North Carolina at Chapel Hill, \\ Chapel Hill, North Carolina, USA.
}

Unlike other picornaviruses, hepatitis A virus (HAV) is cloaked in host membranes when released from cells, providing protection from neutralizing antibodies and facilitating spread in the liver. Acute HAV infection is typified by minimal type I IFN responses; therefore, we questioned whether plasmacytoid dendritic cells (pDCs), which produce IFN when activated, are capable of sensing enveloped virions (eHAV). Although concentrated nonenveloped virus failed to activate freshly isolated human pDCs, these cells produced substantial amounts of IFN- $\alpha$ via TLR7 signaling when cocultured with infected cells. pDCs required either close contact with infected cells or exposure to concentrated culture supernatants for IFN- $\alpha$ production. In isopycnic and rate-zonal gradients, pDC-activating material cosedimented with eHAV but not membranebound acetylcholinesterase, suggesting that eHAV, and not viral RNA exosomes, is responsible for IFN- $\alpha$ induction. pDC activation did not require virus replication and was associated with efficient eHAV uptake, which was facilitated by phosphatidylserine receptors on pDCs. In chimpanzees, pDCs were transiently recruited to the liver early in infection, during or shortly before maximal intrahepatic IFN-stimulated gene expression, but disappeared prior to inflammation onset. Our data reveal that, while membrane envelopment protects HAV against neutralizing antibody, it also facilitates an early but limited detection of HAV infection by pDCs.

\section{Introduction}

Plasmacytoid dendritic cells (pDCs) are "professional" type I IFN-producer cells that play a central role in host antiviral immunity $(1,2)$. Typically present at low frequency in peripheral blood, they migrate to sites of infection and, when activated, produce prodigious quantities of IFN- $\alpha$. Activated pDCs also secrete proinflammatory cytokines, upregulate surface expression of maturation markers, present antigens, and thereby contribute significantly to $\mathrm{CD}^{+} \mathrm{T}$ cell and B cell development $(3,4)$. Thus, these cells effectively bridge innate and adaptive antiviral immunity. pDCs primarily sense viruses via endosomal TLR7 and TLR9 (5), but they can also sense viral nucleic acids in the cytosol (6).

Although early studies suggested that peripheral blood mononuclear cells are stimulated to produce IFN- $\alpha$ more efficiently by enveloped viruses compared with nonenveloped viruses (7), more recent studies indicate that $\mathrm{pDCs}$ are activated by exposure to both types of viruses $(4,8)$. Nonetheless, some picornaviruses (aphthoviruses and some strains of coxsackievirus B) activate

Note regarding evaluation of this manuscript: Manuscripts authored by scientists associated with Duke University, The University of North Carolina at Chapel Hill, Duke-NUS, and the Sanford-Burnham Medical Research Institute are handled not by members of the editorial board but rather by the science editors, who consult with selected external editors and reviewers.

Conflict of interest: The authors have declared that no conflict of interest exists. Submitted: June 12, 2014; Accepted: October 23, 2014.

Reference information: / Clin Invest. 2015;125(1):169-176. doi:10.1172/JCI77527.
pDCs only in the presence of antiviral antibodies, suggesting that uptake of the virus is limiting and requires $\mathrm{Fc}$ receptors $(9,10)$. Internalization thus appears to be critical for sensing of picornaviruses by pDCs, while replication of the viral genome is not always required. Hepatitis A virus (HAV) is an unusual member of the Picornaviridae that has two mature infectious forms, one that is wrapped in host cell membranes (enveloped) and one that is not (11). The enveloped form of the virus (eHAV) is the predominant if not the only form of the virus released into the peripheral circulation during acute hepatitis A, and it is distinct from the more typical nonenveloped "naked" picornaviral virions shed in feces (11). The near absence of type I IFN-stimulated gene (ISG) expression is a striking feature of acute HAV infection in primates (12), calling into question whether $\mathrm{pDCs}$ are capable of sensing the very large amounts of eHAV produced within the liver.

HAV is highly hepatotropic, and infection with HAV typically causes moderate to severe acute inflammatory liver injury. Although it appears incapable of establishing persistent infections even in immunocompromised persons (13), HAV shares common strategies for evasion of innate immune responses with hepatitis $\mathrm{C}$ virus (HCV), also a positive-strand RNA virus, but one that is uniquely capable of establishing long-term persistence in most infected adults. Both HAV and HCV express proteases that degrade mitochondrial antiviral signaling protein (MAVS) and TIR-domain-containing adapter-inducing IFN- $\beta$ (TRIF, also known as TICAM1), key adaptor molecules for retinoic acid-inducible gene I-like (RIG-I-like) receptor (RLR) and TLR3-mediated induction of 

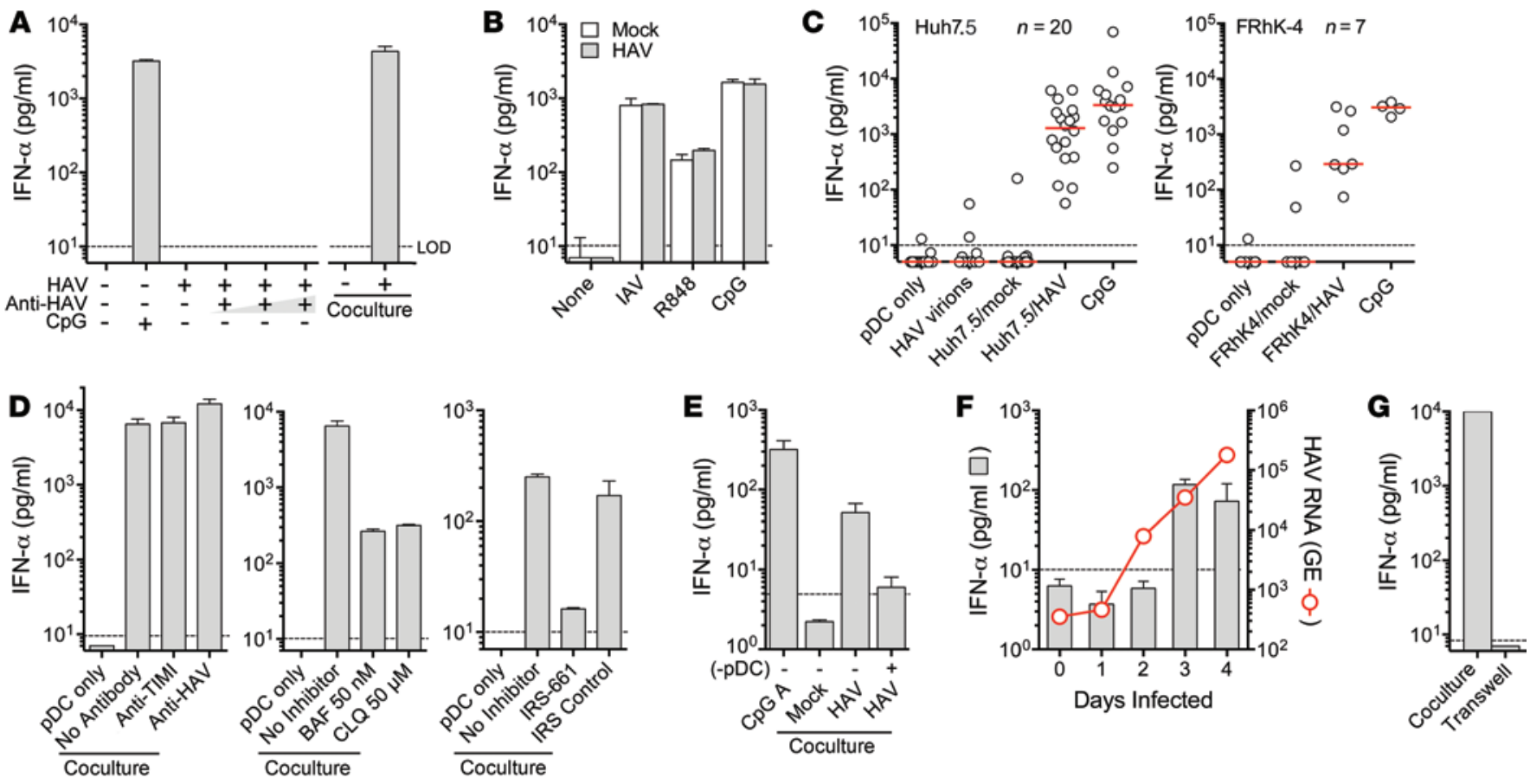

Figure 1. Coculturing with HAV-infected cells induces IFN- $\alpha$ production by pDCs. (A) pDCs $\left(4 \times 10^{5} / \mathrm{ml}\right)$ were exposed to nonenveloped HAV (MOI $\left.=20\right)$, either with or without anti-HAV antibody, or cocultured with HM175/p16 virus-infected cells $\left(1 \times 10^{6} / \mathrm{ml}\right)$. Supernatant IFN- $\alpha$ was measured at 20 hours by ELISA. CpC DNA (ODN2216, $1 \mu \mathrm{M}$ ) was used as a positive control. LOD, limit of detection. (B) pDCs were exposed to influenza A virus (IAV, $6 \mathrm{HA}$ units/ml), R848 $(1 \mu \mathrm{M})$, or $\mathrm{CpG}$ in the presence or absence of HAV. (C) pDCs from multiple donors were exposed to CpG or HAV or cocultured with mock or HAVinfected Huh-7.5 cells or FRhK-4 cells for 20 hours. Red bars indicate median. (D) pDCs and HAV-infected cells were cocultured in the presence of monoclonal anti-HAV antibody or control IgG, following treatment with either bafilomycin A (BAF, $50 \mathrm{nM}$ ) or chloroquine (CLQ, $50 \mu \mathrm{M})$ or $1 \mu \mathrm{M}$ TLR7 antagonist IRS-661 or control IRS. Results are representative of 2 independent experiments. (E) Total or pDC-depleted PBMCs (-pDC) were exposed to $1 \mu M$ CpC A or cocultured with mock or HAV-infected Huh-7.5 cells. (F) pDCs were cocultured with cells infected for increasing numbers of days. Supernatant IFN- $\alpha$ (bars) and intracellular HAV RNA (red line) are shown. GE, genome equivalents. (G) pDCs and HAV-infected cells were cocultured with or without separation by a permeable membrane (pore size $=1 \mu \mathrm{m}$ ). Data represent mean $\pm \mathrm{SEM}$ in $\mathbf{A}, \mathbf{B}$, and $\mathbf{D}-\mathbf{G}$.

type I IFNs (14-17). Despite this, unlike the liver in acute hepatitis A, intrahepatic type I ISG expression is often robust in both acute and chronic hepatitis $C(12,18-20)$. In part, this may be due to the ability of pDCs to sense HCV-infected cells. pDCs are not stimulated to produce IFN- $\alpha$ when incubated with high-titer purified HCV virions, but they are potently activated via TLR7 when cocultured with virus-infected cells (21). This results from short-range exosomal transfer of HCV RNA from virus-infected cells to pDCs in a process dependent upon components of the cellular endosomal sorting complex required for transport (ESCRT) (22). Since the biogenesis of eHAV particles is also dependent upon ESCRT (11) and the intrahepatic abundance of HAV RNA exceeds that of HCV RNA by orders of magnitude during acute infection, despite much lower levels of intrahepatic ISG transcripts (12), we set out to characterize the interactions of enveloped and nonenveloped virions with freshly isolated human pDCs.

\section{Results}

HAV-infected cells, but not purified nonenveloped virions, stimulate human pDCs to produce IFN- $\alpha$. We isolated BDCA $4^{+} \mathrm{pDCs}$ by positive selection from the blood of healthy human donors and characterized the $\mathrm{pDC}$ response to HAV in vitro. pDCs exposed to CpG-A (a TLR9 agonist) produced large quantities of IFN- $\alpha$, but high concentrations of nonenveloped HAV virions failed to stimulate pDCs either in the presence or absence of anticapsid antibody (Figure 1A). The lack of IFN- $\alpha$ production was not due to active suppression of pDCs, since HAV had no effect on IFN- $\alpha$ production induced by CpG-A, R848 (a TLR7 agonist), or influenza A virus (Figure 1B). However, pDCs secreted abundant IFN- $\alpha$ when cocultured with Huh-7.5 hepatoma cells infected with low passage, noncytopathic HAV (ref. 23 and Figure 1A). These results were reproduced with pDCs from multiple donors (Figure 1C). pDCs were also activated when cocultured with infected fetal Rhesus monkey kidney (FRhK-4) cells (Figure 1C). Neither antibody to the putative HAV cellular receptor, $\mathrm{T}$ cell immunoglobulin mucin family member 1 (TIM1, also known as HAVCR1) (24), nor neutralizing anti-HAV antibody blocked this response (Figure 1D), suggesting that it is not induced by nonenveloped HAV virions.

IFN- $\alpha$ production was ablated by coculturing pDCs with infected Huh-7.5 cells in the presence of chloroquine or bafilomycin A1 (Figure 1D, middle), suggesting a requirement for endosomal acidification. Consistent with this, the TLR7-specific inhibitor, IRS-661, but not a control oligonucleotide, markedly blocked IFN- $\alpha$ production (Figure 1D). We confirmed that pDCs were the responsible cell type by demonstrating a marked reduction in IFN- $\alpha$ production when peripheral mononuclear cells were depleted of $\mathrm{BDCA} 4^{+}$cells prior to cocultivation with infected cells 

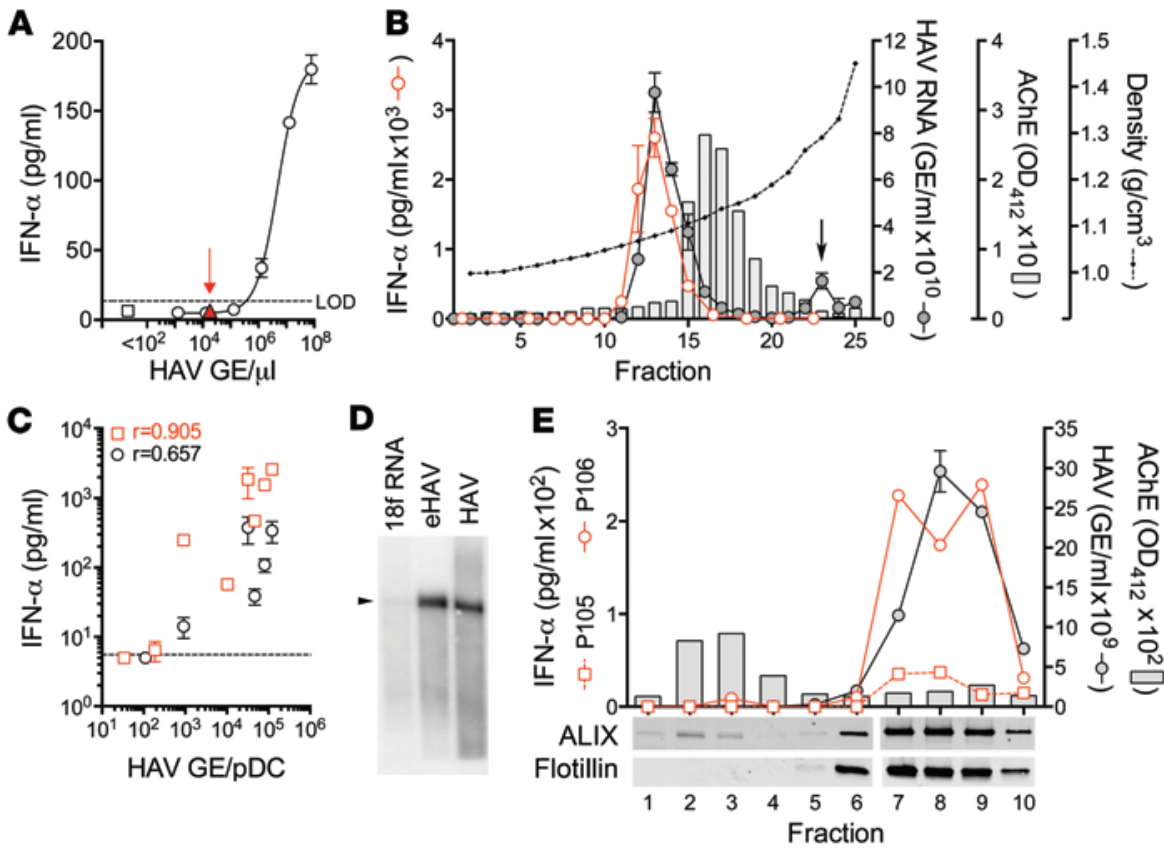

Figure 2. eHAV induces IFN- $\alpha$ production by pDCs. (A) Serial dilutions of concentrated supernatant $\left(100,000-g\right.$ pellet) from HAV-infected cells were mixed with $\mathrm{pDCs}\left(1 \times 10^{6} / \mathrm{ml}\right)$ and incubated for 20 hours. IFN- $\alpha$ levels (mean \pm range in replicate assays) are plotted against HAV RNA content. Unconcentrated supernatant from infected (red arrow and triangle) and mock-infected (white square) cells were tested in parallel. (B) Concentrated supernatant fluids from HAV-infected cells were subjected to isopycnic gradient centrifugation, and individual gradient fractions were incubated with pDCs $\left(1 \times 10^{6} / \mathrm{ml}\right)$. HAV RNA content of fractions was determined by RT-qPCR. IFN- $\alpha$ production shown represents results from 3 donors (mean \pm SEM). AChE activity was measured by enzyme assay. (C) Correlation (Spearman's test) between eHAV content of isopycnic gradient fractions, shown as genome equivalent per pDC, and IFN- $\alpha$ produced (mean \pm range in replicate assays). (D) Northern blot of HAV RNA: full-length in vitro-transcribed HM175/18f HAV RNA, RNA extracted from peak isopycnic gradient fractions containing eHAV, or nonenveloped HAV (mean \pm range in replicate assays). (E) Consecutive gradient fractions containing eHAV from a gradient similar to that in $\mathbf{B}$ were pooled, concentrated, and subjected to rate-zonal ultracentrifugation. Fractions were collected from the top and assessed for HAV RNA content, AChE activity, and pDC stimulating activity (P105 and P106 indicate individual donors) (mean \pm range in replicate assays). Representative results from 1 of 3 independent experiments are shown. Western blots of ALIX and flotillin-1 in the rate-zonal gradient fractions are shown.

(Figure 1E). IFN- $\alpha$ production also correlated with the abundance of HAV RNA in infected cells (Figure 1F). Importantly, pDCs were stimulated only when in close proximity to HAV-infected cells and not when separated by a permeable membrane (Figure 1G). These findings parallel observations with HCV (21), and suggest that the weak intrahepatic ISG responses observed in acute hepatitis A (12) are not due to an inability of pDCs to sense HAV-infected cells.

Enveloped eHAV virions mediate pDC activation. Since highly concentrated preparations of nonenveloped virions do not stimulate pDCs, it is likely that the IFN- $\alpha$ produced by pDCs, when cocultured with infected cells, reflects sensing of enveloped virions. Alternatively, HAV RNA may be transferred to pDCs as cargo within exosomes released by infected cells, as proposed for $\mathrm{HCV}$ (22). Consistent with either hypothesis, a 100-fold concentration of supernatant fluids from infected cells containing predominantly enveloped eHAV particles stimulated pDCs to produce IFN- $\alpha$ (Figure 2A). To resolve the activating material, we subjected concentrated cell culture fluids to ultracentrifugation in isopycnic iodixanol gradients to separate enveloped and nonenveloped virions (11). We identified virions in gradient fractions by RT-qPCR specific for HAV RNA and used an enzyme assay to track acetylcholinesterase (AChE), which is present on exosome membranes $(25,26)$. AChE activity peaked in fractions with a density of $1.12 \mathrm{~g} / \mathrm{cm}^{3}$ (fraction 16), in contrast to eHAV, which banded at $\sim 1.08 \mathrm{~g} / \mathrm{cm}^{3}$ (fraction 13) (Figure 2B). Our previous studies have demonstrated that programmed cell death 6 interacting protein (ALIX, also known as PDCD6IP), an ESCRT-associated protein involved in the biogenesis of both exosomes and eHAV, bands in 2 distinct peaks at these densities in similar gradients $(11,27)$. Remarkably, when incubated with pDCs, the fractions most efficiently triggering IFN- $\alpha$ production were those containing eHAV (Figure 2B). Moreover, the magnitude of IFN- $\alpha$ produced correlated strongly with the abundance of eHAV RNA (Figure 2C), which Northern blot analysis indicated was predominately genome length (Figure 2D). Taken collectively, these data suggest that eHAV, not exosomes containing fragments of HAV RNA, account for most pDC activation. To further resolve the $\mathrm{pDC}$-stimulating activity, isopycnic gradient fractions containing eHAV were subjected to a second round of centrifugation in rate-zonal gradients (25). This allowed for separation on the basis of size and sedimentation velocity rather than density. The AChE activity that cobanded with eHAV in isopycnic gradients sedimented more slowly than eHAV in rate-zonal gradients, peaking in fractions 2-4 rather than fractions 7-9 (Figure 2E). ALIX was present in both membrane fractions, while flotillin-1, which is associated with eHAV as well as exosomes (11), was detected only in the eHAV fractions (Figure 2E). pDC stimulation was largely confined to the eHAV-containing fractions. These results provide further evidence that pDCs are stimulated by eHAV, not more slowly sedimenting exosomes that do not contain encapsidated HAV genomes.

Efficient uptake of eHAV accounts for $P D C$ activation. We considered the possibility that e preferential sensing of eHAV compared with that of HAV by pDCs might reflect greater uptake of enveloped virions compared with that of nonenveloped virions. To test this, we incubated freshly isolated pDCs with equal quantities of gradient-purified eHAV or HAV (quantified by RT-qPCR). The cells were washed extensively at intervals, and RNA was extracted to assess the quantity of cell-associated viral genomes. Substantially greater quantities of eHAV were associated with pDCs compared with nonenveloped HAV after 1 hour (Figure $3 \mathrm{~A})$. Whereas the amount of cell-associated eHAV RNA remained stable or increased slightly over the ensuing 23 hours, the quantity of cell-associated nonenveloped virion RNA progressively decreased. Consistent with this, only pDCs exposed to eHAV pro- 
A

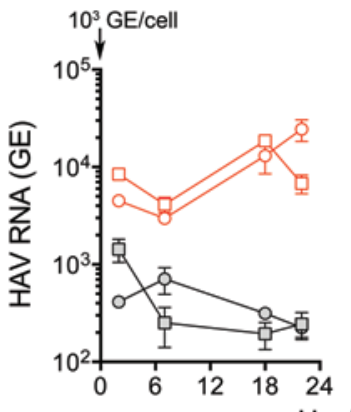

Hrs Exposure

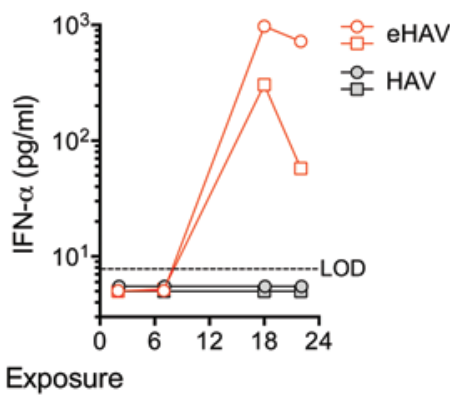

B

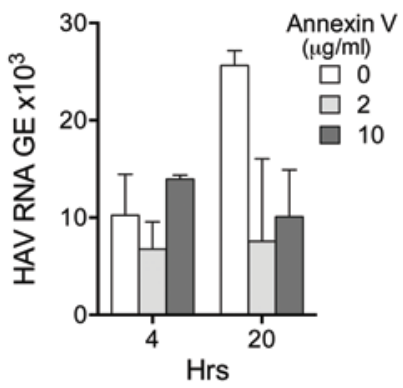

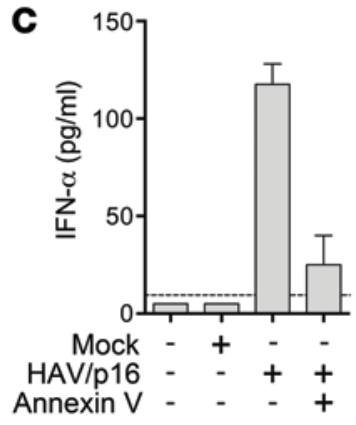
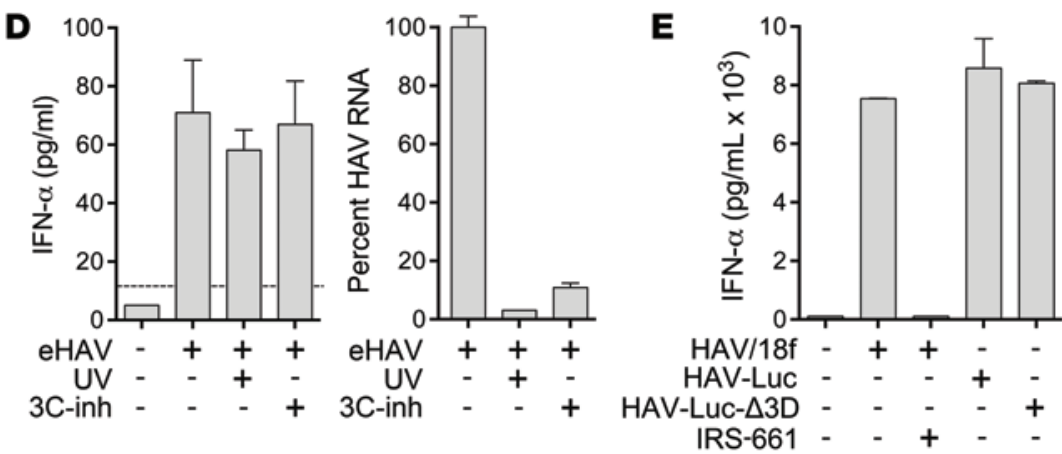

Figure 3. Differential uptake of eHAV versus nonenveloped HAV by pDCs. (A) pDCs were exposed to equal quantities of gradient-purified eHAV or nonenveloped $\mathrm{HAV}$, and cell-associated viral RNA and supernatant IFN- $\alpha$ levels were determined at intervals. Circles and squares represent cells from 2 individual donors, respectively. (B) pDCs were incubated with eHAV in the presence or absence of annexin $\mathrm{V}$ ( 2 or $10 \mu \mathrm{g} / \mathrm{ml}$ ), and cell-associated viral RNA was determined at intervals by RT-qPCR. (C) IFN- $\alpha$ produced by pDCs exposed to concentrated supernatant fluids from mock- or HM175/p16-infected cell cultures in the presence or absence of annexin $V(2 \mu \mathrm{g} / \mathrm{ml})$. (D) IFN- $\alpha$ produced by pDCs exposed to gradient-purified eHAV following inactivation by

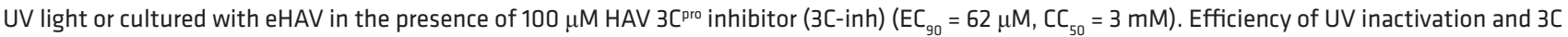
inhibitor treatment. Huh-7.5 cells were infected with UV-inactivated eHAV or nontreated eHAV in the presence of 3C-inh, and cell-associated HAV RNA was measured by RT-qPCR and compared with that in cells infected with untreated HAV at 48 hours. (E) pDCs were transfected with HM175/18f genomic RNA in the presence and absence of IRS-661 or subgenomic HAV-luc RNA or a replication incompetent variant HAV-Luc- $\Delta 3 \mathrm{D}$. Supernatant IFN- $\alpha$ levels were measured by ELISA. Results represent the mean \pm SEM ( $n=2$ or 3 cultures) obtained with pDCs from single donors.

duced IFN- $\alpha$ (Figure 3A). Thus, eHAV is taken up more efficiently by pDCs compared with nonenveloped HAV, a property conferred by the envelope, and this is likely to account for the ability of eHAV to activate pDCs.

HAV does not encode any glycoproteins, and there is no evidence suggesting that viral proteins exist on the surface of eHAV. Since the entry of many enveloped viruses is facilitated by TIM/ TAM family members binding phosphatidylserine (PtdSer) on their surface $(28,29)$ and since TIM1 is constitutively expressed by pDCs (30), we asked whether such an interaction plays a role in eHAV uptake. Indeed, recombinant annexin V (which binds PtdSer) (28) inhibited eHAV uptake by pDCs (Figure 3B). Annexin V also reduced IFN- $\alpha$ production by $\mathrm{pDCs}$ exposed to concentrated supernatant fluids from HAV-infected cell cultures (Figure 3C). However, antibody to TIM1 did not block eHAV uptake (data not shown), suggesting PtdSer receptors other than TIM1 are involved in eHAV uptake. Intriguingly, although TIM1 is considered to be the cellular receptor for nonenveloped HAV (24), nonenveloped virions were not taken up efficiently by pDCs.

HAV genome replication is not required for induction of IFN- $\alpha$ expression by pDCs, as neither UV inactivation nor an inhibitor of the HAV $3 C^{\text {pro }}$ protease (31) diminished this response (Figure $3 \mathrm{D})$. We also found that pDCs could be activated by transfection of synthetic replicon RNA (HAV-Luc) into pDCs and that a replica- tion-incompetent version of the replicon, with a frame-shift mutation in the polymerase coding sequence (HAV-Luc- $\Delta 3 \mathrm{D}$ ), stimulated similar levels of IFN- $\alpha$ production (Figure $3 \mathrm{E}$ ).

pDCs are transiently recruited to the liver during acute HAV infection. Since our data show that pDCs are capable of sensing HAV infection and secrete large amounts of IFN- $\alpha$, we sought to understand why a robust type I IFN response does not occur within the liver during acute hepatitis A. To determine whether pDCs are recruited to the liver, we studied available archived materials from 2 chimpanzees that were previously experimentally infected with HAV (12). Immunofluorescence staining of sections of liver tissue, using both polyclonal and monoclonal antibodies against human C-type lectin domain family 4, member C (BDCA2, also known as CD303 or CLEC4C), a specific marker for pDCs, revealed numerous $\mathrm{BDCA}^{+}$cells within sinusoidal spaces 1 week after virus challenge in both animals (Figure 4). In chimpanzee 4x0293, there was less than $1 \mathrm{BDCA}^{+}$cell per $10^{3}$ nucleated cells prior to intravenous challenge with HAV. This was unchanged 2 days after challenge but increased to $29 \pm 9 \mathrm{BDCA} 2^{+}$cells per $10^{3}$ nucleated cells at 1 week, when intrahepatic expression of ISG15 and IFN-induced protein with tetratricopeptide repeats 1 (IFIT1) mRNA was maximal (12), then fell to 1.6 per $10^{3}$ cells at 2 weeks (Figure $4 \mathrm{~A}$ ). pDC infiltration followed a similar kinetic pattern in chimpanzee 4x0395, but ISG mRNA expression peaked somewhat later, at 2 
A
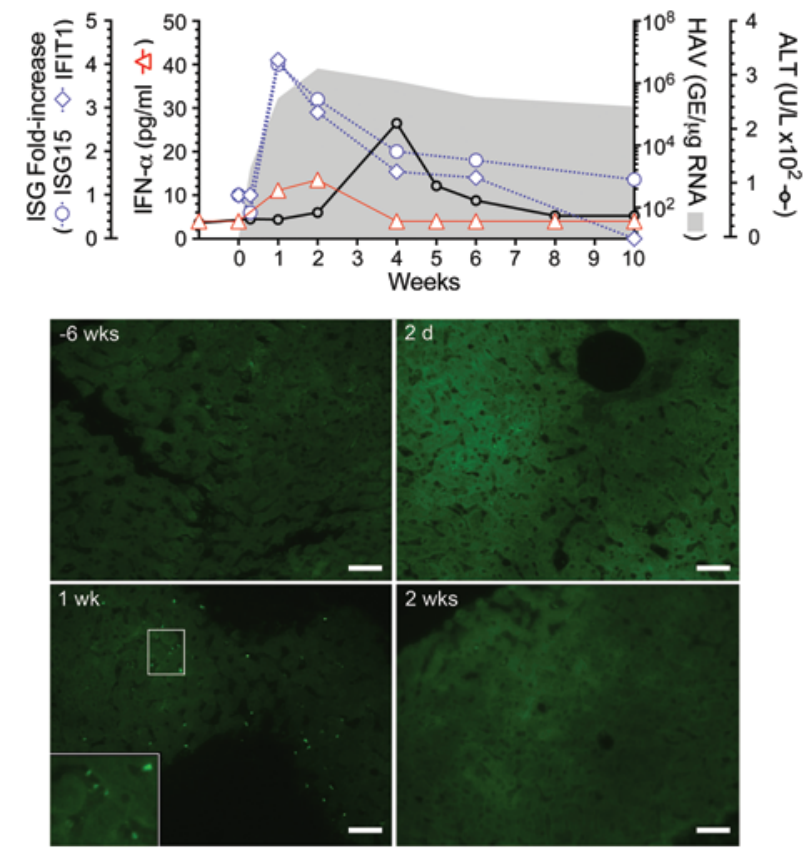

B

\section{$4 \times 0395$}
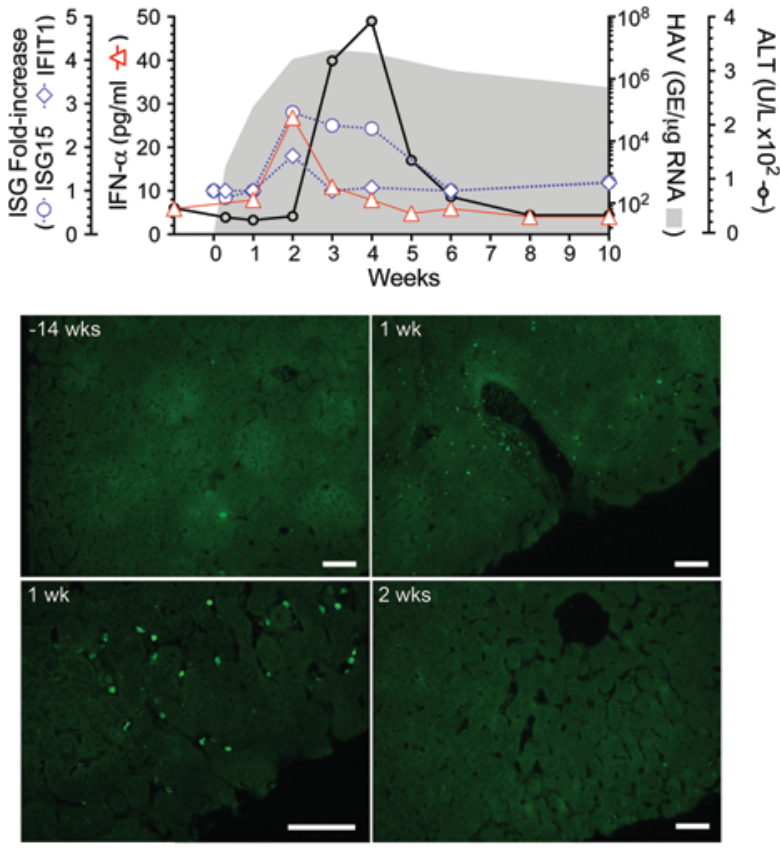

Figure 4. pDCs are transiently recruited to the livers of HAV-infected chimpanzees. (A) Serum IFN- $\alpha$ and changes in intrahepatic ISC15 and IFIT1 mRNA abundance are shown together with levels of intrahepatic HAV RNA in a chimpanzee, 4x0293, experimentally infected with HAV. The ISG15 response and virologic events have been described previously (12). Sections of archived liver specimens collected from chimpanzee $4 \times 0293$ at indicated times prior to and after intravenous challenge with HAV were stained with rabbit polyclonal antibody to human BDCA2. (B) Serum IFN- $\alpha$ and ISC15 and IFIT1 mRNA and ALT activities and intrahepatic HAV RNA in chimpanzee 4x0395 that was similarly infected with HAV (12). Sections of archived liver biopsies from 4x0395 were stained with murine monoclonal antibody 15B to human BDCA2. Scale bars: $200 \mu \mathrm{m}$.

weeks (Figure 4B). Importantly, eHAV was present in the blood of both animals at week $1(11,12)$. Viremia peaked at weeks $2-3$ and then declined rapidly, despite persistence of viral RNA in the liver.

Cytokine ELISA demonstrated the presence of low levels of circulating IFN- $\alpha$ in both animals (Figure 4). This peaked at 14 to $27 \mathrm{pg} / \mathrm{ml} 1$ to 2 weeks after virus challenge, prior to maximal intrahepatic viral RNA abundance or serum ALT elevation (ref. 12 and Figure 4). Sampling intervals in these previous chimpanzee studies were insufficient to determine whether pDC infiltration, serum IFN- $\alpha$ levels, and intrahepatic ISG expression were temporally related. However, the data show that pDCs are recruited to the liver early in HAV infection but disappear many days before viral replication peaks and inflammation develops.

\section{Discussion}

Since HAV infections are almost always controlled and eliminated by host immunity, an understanding of how the virus is sensed by the host provides important insight into a successful antiviral immune response. Data presented here advance our knowledge of the immune response in hepatitis A and indicate that the absence of strong type I ISG expression within the liver relates, at least in part, to the absence of a continuing presence of pDCs, not an inability of pDCs to sense the infection. Although the membrane enveloping eHAV sequesters the viral capsid and protects it from neutralizing antibodies while circulating in the host (11), it enhances the visibility of the virus to pDCs and may account for the minimal type I IFN response observed early in acute infection (12).
The efficient sensing of HAV-infected cells by pDCs (Figure 1C) parallels previous observations with HCV (21). An important distinction, however, is that activation of pDCs results from recognition of enveloped eHAV virions released by these cells (Figure 2) and not viral RNA-laden exosomes as suggested for HCV (22). The preferential sensing of eHAV compared with that of nonenveloped virions by freshly isolated human pDCs reflects distinct interactions of these particles at the level of cell attachment and/ or endocytosis (Figure 3A). The membrane that wraps the capsid in the eHAV virion undoubtedly plays a key role in this process. PtdSer is often present on the membrane of enveloped viruses and promotes cellular attachment and entry of these viruses via interactions with TIM/TAM family proteins $(28,29)$. The partial inhibition of pDC uptake of eHAV by annexin V (Figure 3B) suggests a role for PtdSer, but it remains possible that unknown cellular proteins associated with the membrane contribute to this process. An alternative possibility is that eHAV is internalized by macropinocytosis, a nonspecific fluid-based endocytic mechanism that is constitutively active in pDCs (32).

The inhibition of IFN- $\alpha$ secretion following treatment with IRS-661 suggested that eHAV activates pDCs mainly via TLR7 (Figure 1D), although a minor contribution from the cytosolic pathogen recognition receptors RIG-I and MDA5 cannot be excluded. TLR7 sensing presumably occurs after degradation of the eHAV membrane within a late endosomal/lysosomal compartment (11). How TLR7 then gains access to the RNA that remains fully encapsidated after degradation of the eHAV membrane is 
unclear. Little is known about HAV uncoating and how encapsidated RNA reaches the cytoplasm. The RNA genome of poliovirus (a well-studied picornavirus) is transferred directly across the endosomal membrane into the cytoplasm via an umbilicus that forms as a result of interactions of the capsid with its receptor (33). The HAV genome may be similarly transferred, but recent crystallographic studies of the HAV capsid suggest a fundamental difference in the mechanism of uncoating and raise the possibility that encapsidated RNA is taken up into the cell intact, with disassembly occurring subsequently within the cytoplasm (34). Either way, it is unlikely that HAV RNA is released into the endosomal lumen during entry of the virus. One possibility is that cytosolic HAV RNA traffics to the TLR7 compartment via an autophagy-dependent process (35). Alternatively, a fraction of virions could be degraded in the lysosome, allowing viral RNA to be exposed to TLR7. Yet a third scenario is that nonencapsidated viral RNA may be adventitiously packaged into eHAV during the envelopment of the capsid and that these RNA molecules, embedded between the enveloping membrane and the capsid, are released upon membrane degradation. It is interesting to note, however, that the viral RNA present within the activating eHAV particles was predominantly if not exclusively of genome length (Figure 2D), consistent with it being entirely encapsidated.

The lack of a strong type I ISG response within the liver during acute hepatitis A (12) can be attributed to the absence of pDCs within the liver during the peak phase of the infection coupled with the cleavage of both MAVS and TRIF by HAV proteases $(14,15)$. The signals that result in the initial recruitment of pDCs to the liver early in the course of the infection (Figure 4) are unknown. It is interesting, however, that the pDCs we identified within the liver during the first week of the infection were distributed within the hepatic sinusoids (Figure 4) and not clustered near portal tracts in which inflammatory cells are typically found. The fate of these pDCs and why their numbers are reduced within the liver at later time points are equally uncertain. One possibility is that they undergo conversion to conventional DCs, with reduced type I IFN production (2). Alternatively, they may undergo apoptosis $(36,37)$.

While the early intrahepatic type I IFN response in chimpanzee $4 \times 0293$ correlated well with the presence of pDCs in the liver, the presence of pDCs appears to have preceded intrahepatic ISG expression in chimpanzee 4x0395 (Figure 4). Thus, it is not clear whether the early and weak-type I IFN response results from pDC sensing of the virus or from RLRs or TLR3 prior to full expression of HAV proteases in infected cells. Either way, there is insufficient type I IFN to impair replication of the virus. In mice, T cell priming requires antigen receptor ligation, costimulation, and direct inflammatory stimulation with signal 3 cytokines. Type I IFN is an essential signal 3 cytokine for generation of functional acutephase $\mathrm{CD}^{+}$and $\mathrm{CD} 8^{+} \mathrm{T}$ cells $(38,39)$. The time-limited engagement of pDCs and restricted type I IFN response that accompany HAV infection are sufficient to support the development of robust $\mathrm{CD}^{+} \mathrm{T}$ cell and $\mathrm{B}$ cell responses and to eliminate the virus over time $(12,40)$. However, whether the type I IFN response is sufficient for $\mathrm{CD}^{+} \mathrm{T}$ cell immunity is less certain, because the acutephase HAV-specific response to class I epitopes in these animals was weak and delayed (40).
Prolonged type I IFN signaling can facilitate virus persistence in mice (41). The transient and weak nature of the intrahepatic type I IFN response during acute hepatitis A contrasts sharply with the prolonged, robust response during acute hepatitis $C(12,20)$. pDCs do accumulate in the liver during the chronic phase of HCV infection (42, 43), but whether they infiltrate liver during the acute phase of infection has not been studied. A strong, prolonged pDC response during acute $\mathrm{HCV}$ infection compared with a temporally limited, restricted pDC response during HAV infection could provide a partial explanation for the difference in persistence of these viruses in humans.

\section{Methods}

Reagents and antibodies. Chemical reagents were purchased from Sigma-Aldrich unless otherwise noted. CpG A ODN2216 and R848 were purchased from Invivogen. IRS-661 and a related control oligonucleotide (5'-TCCTGCAGGTTAAGT-3') were synthesized by Integrated DNA Technologies. Influenza A virus (PR8 strain) was obtained from Charles River Laboratory. An HAV $3 C^{\text {pro }}$ protease inhibitor (44) was provided by Bruce Malcolm (Tibotec, Mechelen, Belgium). Human convalescent sera (JC plasma) containing high-titer anti-HAV antibody was collected 90 days after the onset of hepatitis A (11). Rabbit polyclonal and mouse monoclonal antibodies to human BDCA2 were gifts from Liguo Zhang (Institute of Biophysics, Chinese Academy of Sciences, Beijing, China) (45). Monoclonal anti-TIM1 3D1 was a gift from Rosemarie DeKruyff (Boston Children's Hospital, Boston, Massachusetts, USA), and monoclonal anti-HAV K2-4F2 was from Commonwealth Serum Laboratories.

Cells and viruses. Huh-7.5 (46) and FRhK-4 cells (47) were cultured in DMEM supplemented with 10\% FBS, penicillin, and streptomycin. HAV strains HM175/18f and p16 were described previously (11). Hightiter HAV stock was prepared by infecting Huh-7.5 cells at low MOI and freeze thawing the infected cells 3 times to release the virus. Nonenveloped virions were prepared by clarifying lysates by low-speed centrifugation, followed by sonication and extraction with an equal volume of chloroform. Enveloped virions were concentrated from supernatants of HAV-infected culture collected between 2 and 6 weeks after inoculation by differential centrifugation, as described below. Infectious virus titers were determined by a modified infectious focus-forming assay (IR-FIFA) (11).

pDC isolation and stimulation. The BDCA-4 Microbead Kit (Miltenyi Biotec) was used to isolate human pDCs from healthy donor buffy coats purchased from the New York Blood Center. All donations tested negative for hepatitis B virus, HCV, and human immunodeficiency virus. Freshly isolated pDCs (between $2 \times 10^{4}$ and $1 \times 10^{5}$ cells per well, $85 \%-95 \%$ purity) were cultured in flat bottom 96 -well plates in RPMI-1640 medium supplemented with 10\% FBS and penicillin and streptomycin and were exposed to ligands (R848, CpG), gradient fractions, or cocultured with Huh-7.5 or FRhK- 4 cells $\left(1 \times 10^{5}\right.$ cells per well) that were either uninfected or infected with HAV. For Transwell assays, pDCs and Huh-7.5 cells were separated by a membrane with a pore size of $1 \mu \mathrm{M}$ (BD Biosciences). Supernatant fluids were collected at approximately 20 hours unless otherwise indicated. Supernatant IFN- $\alpha$ levels were determined with the human IFN- $\alpha$ ELISA Kit (PBL Interferon Source) following the manufacturer's suggested procedure.

Isopycnic gradient centrifugation of virus. Huh-7.5 cells infected with HAV were cultured in DMEM medium supplemented with $10 \%$ exosome-free FBS (prepared by centrifugation at 100,000 $g$ over- 
night). Cell culture supernatant fluids were concentrated by differential centrifugation $\left(1,000 \mathrm{~g}\right.$ for 10 minutes at $4^{\circ} \mathrm{C}$, followed by centrifugation at 10,000 $\mathrm{g}$ for 30 minutes twice) to remove debris and large vesicles. The clarified supernatant was subjected to ultracentrifugation at $100,000 \mathrm{~g}$ for 1 hour at $4^{\circ} \mathrm{C}$. The pellet was resuspended in PBS, loaded onto an $8 \%$ to $40 \%$ iodixanol gradient (Opti-Prep, Sigma-Aldrich), and centrifuged at $141,000 \mathrm{~g}$ for 48 hours. Fractions were collected from the top of the gradient, and the density of each was determined using a Bausch \& Lomb Abbé refractometer.

Rate-zonal centrifugation. Samples were loaded onto a preformed $6 \%-18 \%$ iodixanol gradient and centrifuged at 250,000 $\mathrm{g}$ in a SW55Ti rotor for 2 hours at $4^{\circ} \mathrm{C}$ in a Beckman ultracentrifuge. Approximately 10 fractions were collected from the top of the gradient.

Enzymatic assay for AChE activity. AChE activity was measured as previously described (25). Briefly, $50 \mu \mathrm{l}$ prewarmed gradient fractions were mixed with $50 \mu \mathrm{l}$ reaction buffer containing $1.25 \mathrm{mM}$ acetylthiocholine and 0.1 mM 5,5-dithio-bis (2-nitrobenzoic acid), and the $\mathrm{OD}_{412}$ was determined at 30-second intervals over a period of $10 \mathrm{~min}$ utes with a Synergy 2 (BioTek) microplate reader.

$H A V$-specific RT-qPCR assay. Total RNA from cells and from culture supernatants or gradient fractions were extracted with the RNeasy Kit and the QIAamp viral RNA Isolation Kit (Qiagen), respectively. HAV RNA was measured by real-time reverse transcription quantitative PCR (RT-qPCR) with a synthetic RNA as standards as previously described (12). Intracellular viral RNA was normalized to total RNA levels.

Indirect immunofluorescence staining for BDCA2. Archived formalin-fixed, paraffin-embedded liver specimens from HAV-infected chimpanzees 4x0293 and 4x0395 (12) were collected prior to December 15,2011 . Sections were sequentially treated with xylene, $100 \%$ ethanol, 95\% ethanol, and 70\% ethanol, followed by antigen retrieval by boiling for 15 minutes. Subsequently, sections were fixed with $4 \%$ paraformaldehyde and permeabilized with $0.5 \%$ Triton-X 100 and then stained using the Tyramide Signal Amplification Kit (Invitrogen) following manufacture's recommendations. Polyclonal and monoclonal anti-BDCA2 antibodies (45) were used to stain for pDCs (see legend for Figure 4). Alexa Fluor 488-conjugated goat anti-rabbit or anti-mouse antibodies (Invitrogen) were used as secondary antibodies. The images were acquired with a Nikon Eclipse Ti-s inverted microscope system with NIS-Elements BR 3.10 acquisition software. The number of BDCA2 ${ }^{+}$cells per $10^{3}$ DAPI-positive nucleated cells was determined by a semiautomated approach using Metamorph 7.1 software (Molecular Devices). The mean number of nucleated cells counted per microscopic field was 1,717 \pm 444 SEM.

Luminex. Luminex analysis for plasma cytokines and chemokines was performed by the Immunology Core Laboratory at the Southwest National Primate Research Center under the supervision of Core Director Luis Giavedoni. Blood samples were collected in tubes containing EDTA, and cytokines and chemokines were measured in a 39-plex assay using a Luminex100 with xMAP multianalyte platform (Millipore).

Statistics. Correlations were assessed using the Spearman nonparametric method. Calculations were made using Prism 6 for Mac OS X (GraphPad Software).

Study approval. The purchase of buffy coats obtained from anonymous blood donors through the New York Blood Center was reviewed by the Institutional Review Board of The University of North Carolina at Chapel Hill and determined not to constitute human subjects research.

\section{Acknowledgments}

We thank Liguo Zhang for providing antihuman BDCA2 antibodies, Bruce Malcolm (Tibotec Inc.) for providing the HAV 3C $\mathrm{C}^{\text {pro }}$ protease inhibitor, Takahiro Masaki for assistance with quantitative microscopy, and Lifang Ping and Will Lovell for technical assistance. This work was supported in part by NIH grants U19AI109965 and R01-AI103083 (to S.M. Lemon). The Southwest National Primate Research Center is supported by an NIH primate center base grant (previously NCRR grant P51 RR13986; currently Office of Research Infrastructure Programs/OD P51 OD011133) and by Research Facilities Improvement Program grants C06 RR 12087 and C06 RR016228 (to R.E. Lanford).

Address correspondence to: Stanley M. Lemon, 8.034 BurnettWomack CB \#7292, The University of North Carolina at Chapel Hill, Chapel Hill, North Carolina, USA. Phone: 919.843.1848; E-mail: smlemon@med.unc.edu. Or to: Zongdi Feng, WA4013, Research II, 700 Children's Drive, The Research Institute at The Nationwide Children's Hospital, Columbus, Ohio 43205-2696, USA. Phone: 614.355.2784; E-mail: Zongdi.Feng@nationwidechildrens.org.

Zongdi Feng's present address is: Center for Vaccines and Immunity, The Research Institute at The Nationwide Children's Hospital, Columbus, Ohio, USA.
1. Gilliet M, Cao W, Liu YJ. Plasmacytoid dendritic cells: sensing nucleic acids in viral infection and autoimmune diseases. Nat Rev Immunol. 2008;8(8):594-606.

2. Reizis B, Bunin A, Ghosh HS, Lewis KL, Sisirak V. Plasmacytoid dendritic cells: recent progress and open questions. Annu Rev Immunol. 2011;29:163-183.

3. Cervantes-Barragan L, et al. Plasmacytoid dendritic cells control T-cell response to chronic viral infection. Proc Natl Acad Sci U S A. 2012;109(8):3012-3017.

4. Deal EM, Lahl K, Narvaez CF, Butcher EC, Greenberg HB. Plasmacytoid dendritic cells promote rotavirus-induced human and murine B cell responses. J Clin Invest. 2013;123(6):2464-2474.
5. Lund J, Sato A, Akira S, Medzhitov R, Iwasaki A. Toll-like receptor 9-mediated recognition of Herpes simplex virus- 2 by plasmacytoid dendritic cells. J Exp Med. 2003;198(3):513-520.

6. Hornung V, et al. Replication-dependent potent IFN- $\alpha$ induction in human plasmacytoid dendritic cells by a single-stranded RNA virus. Jimmunol. 2004;173(10):5935-5943.

7. Feldman SB, Ferraro M, Zheng HM, Patel N, Gould-Fogerite S, Fitzgerald-Bocarsly P. Viral induction of low frequency interferon-alpha producing cells. Virology. 1994;204(1):1-7.

8. Pritchard AL, Carroll ML, Burel JG, White OJ, Phipps S, Upham JW. Innate IFNs and plasmacytoid dendritic cells constrain Th2 cytokine responses to rhinovirus: a regulatory mech- anism with relevance to asthma. JImmunol. 2012;188(12):5898-5905.

9. Guzylack-Piriou L, Bergamin F, Gerber M, McCullough KC, Summerfield A. Plasmacytoid dendritic cell activation by foot-and-mouth disease virus requires immune complexes. Eur J Immunol. 2006;36(7):1674-1683.

10. Wang JP, Asher DR, Chan M, Kurt-Jones EA, Finberg RW. Cutting edge: antibody-mediated TLR7-dependent recognition of viral RNA. Jimmunol. 2007;178(6):3363-3367.

11. Feng $Z$, et al. A pathogenic picornavirus acquires an envelope by hijacking cellular membranes. Nature. 2013;496(7445):367-371.

12. Lanford RE, et al. Acute hepatitis A virus infection is associated with a limited type I 
interferon response and persistence of intrahepatic viral RNA. Proc Natl Acad Sci U S A. 2011;108(27):11223-11228.

13. Martin A, Lemon SM. Hepatitis A virus: from discovery to vaccines. Hepatology. 2006; 43(2):S164-SS72.

14. Qu L, et al. Disruption of TLR3 signaling due to cleavage of TRIF by the hepatitis A virus protease-polymerase processing intermediate, 3CD. PLoS Pathog. 2011;7(9):e1002169.

15. Yang $\mathrm{Y}$, et al. Disruption of innate immunity due to mitochondrial targeting of a picornaviral protease precursor. Proc Natl Acad Sci U S A. 2007;104(17):7253-7258.

16. Meylan E, et al. Cardif is an adaptor protein in the RIG-I antiviral pathway and is targeted by hepatitis C virus. Nature. 2005;437(7062):1167-1172.

17. Li K, et al. and Lemon SM. Immune evasion by hepatitis $\mathrm{C}$ virus NS3/4A protease-mediated cleavage of the TLR3 adaptor protein TRIF. Proc Natl Acad Sci U S A. 2005;102(8):2992-2997.

18. Su AI, et al. Genomic analysis of the host response to hepatitis $\mathrm{C}$ virus infection. Proc Natl Acad Sci U S A. 2002;99(24):15669-15674.

19. Bigger $\mathrm{CB}$, et al. Intrahepatic gene expression during chronic hepatitis $\mathrm{C}$ virus infection in chimpanzees. JVirol. 2004;78(24):13779-13792.

20. Sarasin-Filipowicz M, et al. Interferon signaling and treatment outcome in chronic hepatitis C. Proc Natl Acad Sci U S A. 2008;105(19):7034-7039.

21. Takahashi K, et al. Plasmacytoid dendritic cells sense hepatitis $\mathrm{C}$ virus-infected cells, produce interferon, inhibit infection. Proc Natl Acad Sci U S A. 2010;107(16):7431-7436.

22. Dreux M, et al. Short-range exosomal transfer of viral RNA from infected cells to plasmacytoid dendritic cells triggers innate immunity. Cell Host Microbe. 2012;12(4):558-570.

23. Jansen RW, Newbold JE, Lemon SM. Complete nucleotide sequence of a cell culture-adapted variant of hepatitis A virus: comparison with wild-type virus with restricted capacity for in vitro replication. Virology. 1988;163(2):299-307. 24. Feigelstock D, Thompson P, Mattoo P, Zhang
Y, Kaplan GG. The human homolog of HAVcr-1 codes for a hepatitis A virus cellular receptor. JVirol. 1998;72(8):6621-6628.

25. Cantin R, Diou J, Belanger D, Tremblay AM, Gilbert C. Discrimination between exosomes and HIV-1: purification of both vesicles from cell-free supernatants. Jimmunol Methods. 2008;338(1):21-30.

26. Asea A, et al. Heat shock protein-containing exosomes in mid-trimester amniotic fluids. J Reprod Immunol. 2008;79(1):12-17.

27. Bissig C, Gruenberg J. ALIX and the multivesicular endosome: ALIX in Wonderland. Trends Cell Biol. 2014;24(1):19-25.

28. Moller-Tank S, Kondratowicz AS, Davey RA, Rennert PD, Maury W. Role of the phosphatidylserine receptor TIM-1 in enveloped-virus entry. JVirol. 2013;87(15):8327-8341.

29. Morizono K, et al. The soluble serum protein Gas6 bridges virion envelope phosphatidylserine to the TAM receptor tyrosine kinase Axl to mediate viral entry. Cell Host Microbe. 2011;9(4):286-298.

30. Xiao S, et al. Tim-1 stimulation of dendritic cells regulates the balance between effector and regulatory T cells. Eur JImmunol. 2011;41(6):1539-1549.

31. Malcolm BA, et al. Peptide aldehyde inhibitors of hepatitis A virus $3 \mathrm{C}$ proteinase. Biochemistry. 1995;34(25):8172-8179.

32. Mercer J, Helenius A. Virus entry by macropinocytosis. Nat Cell Biol. 2009;11(5):510-520.

33. Strauss M, Levy HC, Bostina M, Filman DJ, Hogle JM. RNA transfer from poliovirus $135 \mathrm{~S}$ particles across membranes is mediated by long umbilical connectors. J Virol. 2013;87(7):3903-3914.

34. Wang $X$, et al. Hepatitis A virus and the origins of picornaviruses [published online ahead of print October 19, 2014]. Nature. doi:10.1038/ nature13806.

35. Lee HK, Lund JM, Ramanathan B, Mizushima $\mathrm{N}$, Iwasaki A. Autophagy-dependent viral recognition by plasmacytoid dendritic cells. Science. 2007;315(5817):1398-1401.

36. Swiecki M, Wang Y, Vermi W, Gilfillan S, Schreiber RD, Colonna M. Type I interferon neg- atively controls plasmacytoid dendritic cell numbers in vivo. J Exp Med. 2011;208(12):2367-2374.

37. Thomas JM, et al. Differential responses of plasmacytoid dendritic cells to influenza virus and distinct viral pathogens. J Virol. 2014;88(18):10758-10766.

38. Welsh RM, Bahl K, Marshall HD, Urban SL. Type 1 interferons and antiviral CD8 T-cell responses. PLoS Pathog. 2012;8(1):e1002352.

39. Wilson EB, Brooks DG. Decoding the complexity of type I interferon to treat persistent viral infections. Trends Microbiol. 2013;21(12):634-640.

40. Zhou Y, et al. Dominance of the CD4(+) Thelper cell response during acute resolving hepatitis A virus infection. JExp Med.2012;209(8):1481-1492.

41. Odorizzi PM, Wherry EJ. Immunology. An interferon paradox. Science. 2013;340(6129):155-156.

42. Lau DT, et al. Interferon regulatory factor-3 activation, hepatic interferon-stimulated gene expression, and immune cell infiltration in hepatitis $\mathrm{C}$ virus patients. Hepatology. 2008;47(3):799-809.

43. Velazquez VM, Hon H, Ibegbu C, Knechtle SJ, Kirk AD, Grakoui A. Hepatic enrichment and activation of myeloid dendritic cells during chronic hepatitis C virus infection. Hepatology. 2012;56(6):2071-2081.

44. Bergmann EM, et al. Crystal structure of an inhibitor complex of the $3 \mathrm{C}$ proteinase from hepatitis A virus (HAV) and implications for the polyprotein processing in HAV. Virology. 1999;265(1):153-163.

45. Li G, et al. Plasmacytoid dendritic cells suppress HIV-1 replication but contribute to HIV-1 induced immunopathogenesis in humanized mice. PLoS Pathog. 2014;10(7):e1004291.

46. Blight KJ, McKeating JA, Rice CM. Highly permissive cell lines for subgenomic and genomic hepatitis C virus RNA replication. J Virol. 2002;76(24):13001-13014.

47. Hopps HE, Bernheim BC, Nisalak A, Tjio JH, Smadel JE. Biologic characteristics of a continuous kidney cell line derived from the African green monkey. JImmunol. 1963;91:416-424. 